Revue de droit comparé du travail et de la sécurité sociale

3 | 2018

La participation des travailleurs dans la grande entreprise privée et publique

\title{
Le droit du travail parmi les droits civils et politiques ? Réflexions à propos de la participation des travailleurs au gouvernement de l'entreprise
}

Isabelle Ferreras

\section{OpenEdition}

Journals

Édition électronique

URL : https://journals.openedition.org/rdctss/1879

DOI : $10.4000 /$ rdctss. 1879

ISSN : 2262-9815

Éditeur

Centre de droit comparé du travail et de la sécurité sociale

Édition imprimée

Date de publication : 1 novembre 2018

Pagination : 28-37

ISSN : 2117-4350

Référence électronique

Isabelle Ferreras, « Le droit du travail parmi les droits civils et politiques ? Réflexions à propos de la participation des travailleurs au gouvernement de l'entreprise », Revue de droit comparé du travail et de la sécurité sociale [En ligne], 3 | 2018, mis en ligne le 01 novembre 2021, consulté le 13 novembre 2021. URL : http://journals.openedition.org/rdctss/1879; DOI : https://doi.org/10.4000/rdctss.1879

\section{(c) $(1) \odot$}

Revue de droit comparé du travail et de la sécurité sociale est mise à disposition selon les termes de la Licence Creative Commons Attribution - Pas d'Utilisation Commerciale - Pas de Modification 4.0 International. 


\title{
LE DROIT DU TRAVAIL PARMI LES DROITS CIVILS ET POLITIQUES ? RÉFLEXIONS À PROPOS DE LA PARTICIPATION DES TRAVAILLEURS AU GOUVERNEMENT DE L'ENTREPRISE ${ }^{1}$
}

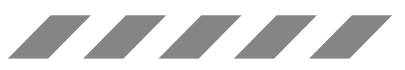

\begin{abstract}
Working from the perspective of a comprehensive and critical sociology of contemporary work, and a companion critical political theory of the firm, this paper defends the following hypothesis: it is useful to consider labor law as directly related to civil and political rights and not to a category of rights, derived, secondary or additional to this first set of rights.

\section{KEY WORDS : Worker Participation, Firm as a Political Entity, Corporate Governance, Public} Space, Contradiction Capitalism / Democracy.
\end{abstract}

\section{RÉSUMÉ}

Au départ d'une sociologie compréhensive et critique du travail et d'une théorie politique de l'entreprise dont il convient de l'équiper, il est pertinent de considérer l'hypothèse suivante : dans une perspective critique et reconstructive, il est temps d'envisager le droit du travail comme émargeant directement des droits civils et politiques et non pas d'une catégorie de droits, dérivés, secondaires ou supplémentaires.

MOTS CLÉS : Participation des travailleurs, entreprise comme entité politique, gouvernement de l'entreprise, espace public, contradiction capitalisme/démocratie.

1 Ce texte a été préparé dans le contexte de deux événements scientifiques : une communication lors des journées d'étude internationales organisées dans le cadre du projet de partenariat CRIMT Institutional Experimentation for Better Work par le Comptrasec à I'Université de Bordeaux les 21 et 22 septembre 2017, portant sur I'« Analyse comparée des dispositifs légaux et/ou conventionnels de participation des travailleurs à la gestion dans la grande entreprise privée et publique », et le colloque portant sur "Les normes du droit du travail : approche interdisciplinaire » organisé par Alexis Cukier (CIPH, Sophiapol, Université Paris Nanterre), Luca Paltrinieri (CIPH, EA Philosophie des normes, Université de Rennes 1) et Vincent Bourdeau (Logiques de l'Agir, Université de Franche-Comté) qui s'est tenu les 14 et 15 décembre 2017 à Paris. L'auteure en remercie les organisateurs. 


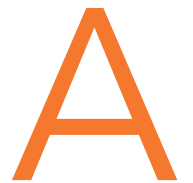

u départ d'une sociologie compréhensive et critique du travail et d'une théorie politique de l'entreprise dont il convient de l'équiper ${ }^{2}$, il est pertinent de considérer l'hypothèse suivante : dans une perspective critique et reconstructive ${ }^{3}$, il est temps d'envisager le droit du travail comme émargeant directement des droits civils et politiques et non pas d'une catégorie de droits, dérivés, secondaires ou supplémentaires. Aujourd'hui, indexée au niveau de développement caractérisant une société ${ }^{4}$, la conception de trois stades de la citoyenneté selon la pensée canonique de T.H. Marshall ${ }^{5}$ (1950) tend pourtant toujours à nous faire penser les enjeux liés au droit des investisseurs en travail en ces termes - dérivés, secondaires ou supplémentaires. Etudiant I'histoire de l'Angleterre, Marshall identifie en effet une évolution graduelle de la conception des droits, allant des droits " premiers ": les droits civils conquis au XVIII'ème, passant par les droits politiques au XIX ${ }^{\mathrm{ème}}$, pour mener

2 Notre agenda de recherches vise à poser les bases d'une telle démarche. Voir I. Ferreras Critique politique du travail. Travailler à l'heure de la société des services. Paris. Les Presses de Science Po, 2007. Gouverner le capitalisme ? Pour le bicamérisme économique. Paris. Presses universitaires de France, 2012. Firms as Political Entities. Saving Democracy through Economic Bicameralism. Cambridge/New York City: University Press, 2017. Le présent texte s'appuie sur les développements proposés dans ces trois ouvrages et ne peut bien évidemment, dans le présent format, fournir tous les développements nécessaires. L'auteur remercie le lecteur de bien vouloir s'y référer pour plus de précisions.

3 Loin du droit positif, la présente contribution, s'appuyant principalement sur la sociologie et la philosophie politique, s'inscrit dans un cadre épistémologique qui cherche, à la suite des travaux de l'Ecole de Francfort et singulièrement ceux d'Habermas (J. Habermas, Jürgen. Ecrits politiques. Paris. Cerf, 1990) visant l'émancipation comme l'intérêt de connaissance spécifique aux sciences critiques, à identifier les « intuitions critiques » (I. Ferreras, Gouverner le capitalisme? Pour le bicamérisme économique. Paris. Presses universitaires de France, 2012) émancipatrices des acteurs et de les renforcer au travers d'une démarche de type reconstructive, entendue dans un sens proche de l'éthique « reconstructive " proposée par Jean-Marc Ferry, (L'Éthique reconstructive, Paris, Le Cerf, 1996). La conception critique du droit qui l'anime est proche de la philosophie du droit avancée par Walter Benjamin (Critique de la violence, Paris, Payot, 2018 (1921) qui voyait dans le droit l'expression d'une sédimentation d'un rapport de forces sociales, et de manière plus contemporaine, le mouvement des Critical legal studies en droit qui cherche aussi à penser le droit comme une construction sociale qui peut potentiellement servir d'instrument d'émancipation.

4 Entendu, dans une compréhension sociologique, au sens de système social, et non pas du contrat relevant du droit des sociétés. La polysémie est néanmoins pleine de sens dans le contexte qui nous occupe ici, la société devenant un " concept nomade » des plus intéressants à suivre au travers des différents champs disciplinaires.

5 T.H. Marshall, Citizenship and Social Class: And Other Essays. Cambridge University Press, 1950. 
enfin aux droits sociaux au XXème. Marshall envisageait ces couches de droit se sédimentant les unes aux - voire, sur - les autres, permettant d'approfondir ainsi un exercice de plus en plus riche de la citoyenneté. Ce séquençage historique rencontre toutefois une limite importante lorsqu'il est mobilisé dans le contexte d'une discussion sur le droit du travail : il laisse croire que les droits dits sociaux sont l'essentiel de ce qui se joue dans le domaine économique.

Or, le droit du travail est mieux compris comme relevant d'une quête de reconnaissance de droits politiques dans le monde du travail lui-même. En ce qu'il contribue à l'occulter, le séquençage de Marshall rencontre de manière non explicite - et renforce - la puissante partition héritée du libéralisme entre champs du politique et de l'économique. Au libéralisme politique, la philosophie du pouvoir dans le champ identifié comme politique, au libéralisme économique la philosophie (du pouvoir) pertinente pour le champ économique. D'un côté, l'égalité d'êtres libres et égaux, de l'autre, la liberté de l'entrepreneur et celle de consentir librement à un contrat de travail -qui signe... la fin de la liberté et de l'égalité, place au principe de subordination.

Il nous faut rompre avec une conception des droits du travail comme relevant de cette troisième vague car elle perpétue la situation d'impasse à laquelle cette partition nous mène : à savoir considérer comme deux sphères d'action totalement distinctes, pas seulement au XVIIIème, XIXème, ou XXème siècle mais aujourd'hui même : les enjeux d'une sphère publique démocratique, pensés bien à l'écart des pratiques économiques, et inversement. Or, aujourd'hui, l'économie fait pleinement partie de l'espace public de nos sociétés démocratiques et continuer à ne pas l'envisager, en refusant de faire des travailleurs des citoyens au travail, nous plonge dans une situation de crise grave. C'est in fine la viabilité même du projet démocratique qui se trouve ici posée 6 .

\section{I - LA CONTRADICTION CAPITALISME/DÉMOCRATIE}

Cette impasse, dans une lecture d'inspiration marxienne ${ }^{7}$, peut être éclairée comme relevant de la situation de contradiction entre capitalisme et démocratie. Aujourd'hui, nombreux sont ceux qui sentent combien le sens de nos démocraties politiques fondées sur un idéal d'égalité est en train de perdre de sa valeur à mesure que le principe structurant le capitalisme ne fait que se renforcer et étendre son influence. Ce principe - capitaliste, résumons-le comme suit : il vise à n'accorder les droits politiques qu'aux seuls détenteurs de capital. Le principe du capitalisme est bien politique et non économique stricto sensu :

6 I. Ferreras, Firms as Political Entities. Saving Democracy through Economic Bicameralism. Cambridge/New York City: University Press, 2017.

7 I. Ferreras, Critique politique du travail. Travailler à l'heure de la société des services. Paris. Les Presses de Science Po, 2007. 
il identifie qui a le droit d'être représenté dans le gouvernement de l'entreprise commune. Le principe capitaliste est clair : seuls les détenteurs de capital participent à ce gouvernement de l'entreprise, y compris du travail. Pourtant, les travailleurs sont également reconnus comme des citoyens, "libres et égaux en dignité et en droits $»^{8}$. Une si grande contradiction entre l'égalité qui fonde l'idéal démocratique et le monopole du pouvoir accordé aux seuls apporteurs de capital ne peut que produire une contradiction de plus en plus flagrante à mesure que, d'une part, les citoyens affirment leur attente de pouvoir peser sur le destin commun, à commencer par leur propre destin (le rejet du "système » et recours aux populistes de tous bords qui se donnent actuellement à voir dans les élections en Occident est à comprendre ainsi), affirmant ainsi la valeur du principe démocratique, tout en voyant (suite aux transformations du capitalisme à présent financiarisé et globalisé, émancipé des contrôles et frontières étatiques) les apporteurs de capital contrôler des parts croissantes de la vie sociale, culture, santé, éducation, etc. jusqu'à, potentiellement, la vie politique elle-même. Dans cette perspective, les USA sont à considérer comme un cas limite, où la contradiction se résout par la victoire du principe capitaliste sur le principe démocratique, y compris dans le champ politique?.

Aux yeux de Marx, les salariés se trouvaient dans une situation d'hétéronomie ${ }^{10}$ - signant leur aliénation. En cela, malgré les droits du travail acquis en 150 ans de luttes syndicales, la situation n'a pas fondamentalement changé. En effet, les salariés ne déterminent toujours pas les fins qu'ils poursuivent par leur travail ni ne participent à déterminer les normes qui de facto s'imposent à eux dans le processus-même de travail. Ce qui a changé par contre, en autant de décennies de capitalisme, c'est l'approfondissement de la culture démocratique. Aujourd'hui, même le plus ignorant, le moins qualifié des salariés, partage ce fonds culturel dans les sociétés démocratiques stabilisées, marqué par l'attente de la reconnaissance de son égalité en dignité et en droit.

8 Ainsi l'Article 1 de la Déclaration universelle des Droits de l'Homme adoptée par l'Assemblée générale des Nations-Unies en 1948 sanctionne le fait que : «Tous les êtres humains naissent libres et égaux en dignité et en droits ॥. II s'inspire directement de la Déclaration des droits de l'homme et du citoyen de 1789 et sanctionne l'idéal de l'individualisme libéral comme fond culturel commun à l'Occident à tout le moins - c'est cet espace géopolitique des démocraties capitalistes qui nous occupe ici.

9 Voir A. Winkler, We the Corporations. How American Businesses Won their Civil Rights. NYC: Norton \& Cie, 2018.

10 Venant de hetero-nomos : qui dépend des lois fixées par autrui, terme qui s'oppose à autonomie, qui obéit à sa propre loi. 


\section{II - LE TRAVAIL, PORTEUR DE L'INTUITION CRITIQUE DE LA JUSTICE DÉMOCRATIQUE}

A l'occasion du passage de l'économie industrielle aux services, l'entrée du client sur le lieu de travail permet d'approfondir la logique " politique » du travail11, au travers de la mobilisation par le travailleur de ses propres conceptions sur le juste et l'injuste dans le cadre d'une inscription collective - ce qui constitue l'expérience du travail contemporain, à I'heure de la flexibilité. De plus, nous assistons avec l'économie de services à un approfondissement de la publicisation des milieux de travail, en raison de l'entrée des clients au cœur de l'espace du travail.

Nous avons proposé de parler du caractère " public » du travail contemporain pour rendre compte de cette réalité nouvelle: de par la présence de ce tiers qu'est le client (ni collègue, ni supérieur hiérarchique - les deux figures de l'autre du monde industriel), l'expérience du travail est aujourd'hui l'occasion, incontournable, de la mobilisation des normes conventionnelles de l'espace public des démocraties libérales avancées, au premier rang desquelles se trouve la convention d'égale dignité. Au cœur du monde industriel, les témoignages abondent, l'ouvrier était touché par le caractère injuste des différentes situations vécues au travail ${ }^{12}$. Mais personne, au quotidien, ne venait lui «mettre sous le nez », lui rappeler de manière continue les normes d'égalité et d'autonomie collective portées par l'idéal démocratique dans la sphère publique. Au travail, dans l'univers domestique de l'usine, le contremaître le traitait « comme un chien » ? C'était la norme du travail - ressentie comme injuste, certes, mais qui s'imposait à tous dans l'espace clos de l'économie, tenu bien à l'écart de l'espace public ${ }^{13}$. Dans l'espace ouvert de l'économie des services, c'est au cœur de leur travail que les travailleurs sont confrontés à la figure nouvelle du client, ce tiers, cet autre citoyen, qui agit pour le salarié comme rappel incessant de son attente d'égalité, support de mobilisation des conventions typiques de l'espace public démocratique, dans l'univers du travail.

Nous avons proposé de comprendre que cette confrontation devient en quelque sorte inéluctable car le salarié, confronté au client comme à cet autre de l'espace public, égal en dignité et en droits, ne peut qu'attendre (espérer...) que ce dernier lui reconnaisse lui aussi son égale dignité. Et partant de là, par un effet de contagion, ces attentes normatives de forte puissance se reportent vis-à-vis de la hiérarchie et du milieu de travail en général. La culture démocratique de l'égalité de l'individu s'approfondissant et le travail des services faisant apparaître au cœur du travail l'autrui de la sphère publique, le vécu du travail comme une expérience proprement politique animée par cette attente de justice démocratique, est devenu aujourd'hui incontournable; c'est ce que nous avons proposé d'appeler l'« intuition critique de la justice démocratique » au travail.

11 I. Ferreras, Critique politique du travail. Travailler à l'heure de la société des services. Paris. Les Presses de Science Po, 2007.

12 A. Faure, J. Rancière (ed.), La parole ouvrière. Paris. La Fabrique, 2007.

13 Espace clos qui portait donc bien son nom, oikos-nomos en grec : la loi de la maison. Le maître étant le domus au service duquel le travailleur se met, à l'image d'un domestique à qui il ne vient pas même l'idée de remettre en cause les décisions de celui qui gouverne. Aujourd'hui, l'économie n'a plus rien à voir avec cet oikos. Au contraire, elle a tout à voir avec l'espace public. Voir I. Ferreras supra note 10. 
Ce vécu approfondit la contradiction capitalisme/démocratie qui se déploie comme tension structurant l'expérience du salariat en démocratie capitaliste : dominé par le régime domestique au travail, subordonné au donneur d'ordre capitaliste et en même temps, baigné dans une culture et une attente démocratique. Le client agit comme un révélateur des tensions entre l'aspiration démocratique à la reconnaissance de l'égale dignité démocratique - au plein sens culturel, tel que l'avait constaté Tocqueville en Amérique - et la négation de celle-ci par le régime de travail de type domestique qui organise toujours la mise au travail dans l'entreprise capitaliste : le client, souvent malgré lui, agit comme support à l'entrée de la culture de l'espace public démocratique dans l'entreprise.

\section{III - L'ENTREPRISE, ENTITÉ POLITIQUE-PIVOT DE LA CONTRADICTION : À DÉMOCRATISER}

A cette lumière, on comprend mieux pourquoi il faut considérer l'entreprise comme institution pivot de cette contradiction capitalisme/démocratie, qui culmine en ce début de XXlème siècle. L'entreprise, en effet, est l'entité au sein de laquelle se vit de manière aiguë cette contradiction majeure dans laquelle nos " démocraties capitalistes " ${ }^{14}$ plongent les individus qui la composent : tiraillés entre le monopole du pouvoir concédé aux seuls apporteurs en capital dans leur vie de travail et l'aspiration à l'égalité démocratique. C'est dès lors au développement d'une théorie politique de l'entreprise, capable de proposer une alternative analytique à la théorie économique de l'entreprise qu'il faut s'atteler. Car rangée au rang d'attribut de la société anonyme, l'entreprise apparaît à l'arrière-plan, nécessaire, de la société qui détient le pouvoir, ce que Robé appelle « construire l'entreprise autour de la société ${ }^{15}$. Du point de vue d'une théorie politique, la structure de gouvernement d'une telle entité, aussi vaguement définie, est une structure monocamérale de gouvernement où les représentants des apporteurs en capital se comportent en despotes au sens où l'entendait Aristote -le maître incontestable, commandant de manière unilatérale l'esclave, dans le contexte domestique ${ }^{16}$. La Chambre des représentants des apporteurs en capital - le Conseil d'administration - occupe le pouvoir législatif et impose son régime domestique à l'ensemble de l'entité concernée, au travers des liens juridiques du droit du travail et du droit commercial qu'elle entretient avec toute sa chaîne de valeur (contrats de soustraitance ou de fourniture).

14 J. Cohen, J. Rogers On Democracy. Toward a Transformation of American Society. New York, Penguin Books, 1983.

15 Notre traduction J.Ph. Robé, "The Legal Structure of the Firm", Accounting, Economics, and Law, 2011, 1(5), 86 p.

16 C'est-à-dire bien l'oikos, d'où vient, comme il l'a été rappelé, le terme "économie", qui vise en effet la loi de la maison, les règles du foyer. 


\section{IV - SORTIR DU DESPOTISME}

La question qui se pose, une nouvelle fois aujourd'hui, est celle de la sortie du despotisme. L'Occident a une longue connaissance en la matière d'identification des despotes. Sous des habits économiques - celui de l'entreprise contrôlée par la société anonyme, l'identification se fait plus difficile. Cependant, la reconnaissance de la propriété - privée - des parts des apporteurs en capital dans la société anonyme (ou toute autre forme de structuration juridique du capital) ne peut empêcher de penser l'architecture des pouvoirs spécifique à la vie de l'entreprise, dans une perspective de démocratisation. L'entreprise est ainsi mieux comprise comme l'« entité politique ${ }^{17}$ qui est définie par les rapports de pouvoir qui animent les relations entre travail et capital; dit autrement : la combinaison d'une structuration juridique du capital, via le droit des sociétés, et d'une structuration institutionnelle encore trop mal définie du travail - via l'amorce à déployer que constitue le droit du travail, et de manière particulièrement significative le droit à la négociation collective et les instances construites sous la pression des travailleurs de type comité d'entreprise ou délégation syndicale.

L'entreprise se trouve confrontée à la question politique classique de la transition du despotisme au gouvernement représentatif, c'est-à-dire une transition de la situation d'hétéronomie qui caractérise la mise au travail dans des conditions capitalistes au droit de tous les investisseurs impliqués de peser sur le gouvernement qui les concerne - et ainsi de les équiper institutionnellement des moyens d'exercice d'une autonomie collective dans un contexte démocratique, au sens de Castoriadis ${ }^{18}$. Cette réflexion sur l'entreprise ne peut donc être arrimée au seul droit de la société et doit dépasser les limites d'un principe de subordination au sens classique tel qu'entendu en droit du travail. Au contraire, elle cherche à prendre sérieusement en considération la dynamique de pouvoir, et donc le potentiel de conflictualité permanent, qui anime les relations qui constituent l'entreprise. En approchant alors l'entreprise, non plus comme une simple organisation économique, saisie par un ensemble de calculs guidés par la seule rationalité instrumentale, mais comme une entité politique à part entière, travaillée par des relations de pouvoir multiples, dont certains aspects seulement sont mesurés au travers du médium monétaire et d'indicateurs quantitatifs.

17 I. Ferreras, Firms as Political Entities. Saving Democracy through Economic Bicameralism. Cambridge/New York City: University Press, 2017.

18 C. Castoriadis, L'institution imaginaire de la société. Paris. Seuil, 1975. 
Durant tout le XXème siècle, les dispositions prises au sein des démocraties capitalistes ont visé à ouvrir le droit pour les travailleurs de participer à la gestion du travail et/ou de l'entreprise. II importe de marquer ici une distinction entre gouvernement et gestion (ou gouvernance $)^{19}$. Gouverner consiste à être associé à une véritable délibération doublée d'une négociation sur les termes de l'échange - économique, donnant lieu à une discussion sur ses finalités et ses moyens ${ }^{20}$. C'est ce qui correspond à l'idée de faire place aux travailleurs dans le gouvernement de l'entreprise. La gestion se contente de marchander (bargain) les moyens de la mise en œuvre d'un projet défini par ailleurs (hétéronomie...), en amont, par les apporteurs en capital. C'est ce qui correspond à l'idée de participer à la gestion de l'entreprise. Dans la délibération, les termes de l'échange sont politiques, ils touchent aux conceptions sur la justice et concernent les finalités de l'activité ; dans la négociation-marchandage, ils se déploient selon un échange quantifié terme à terme : salaire, heures de travail, horaires, cadences contre subordination, productivité et ordre social. Gouverner touche aux finalités en même temps qu'à la poursuite pratique de ces fins; gérer concerne la mise en œuvre d'un cadre préétabli, décidé à la suite d'une délibération qui s'est tenue au préalable, par d'autres acteurs habilités que les investisseurs en travail et/ou leurs représentants. Gouverner se joue dans les coordonnées de l'espace public démocratique sur base de la reconnaissance de l'égalité de tous les participantscontributeurs, gérer s'inscrit encore dans la sphère privée où ceux qui sont en position de force peuvent imposer leurs conditions aux termes de l'échange. Une longue suite de dispositifs, dont les études de gestion ont fait leur objet, a visé à impliquer les travailleurs dans la gestion de l'entreprise, afin de rendre son fonctionnement et sa production plus efficace ${ }^{21}$. Au contraire, l'enjeu d'une réflexion critique sur le travail est de poser aujourd'hui la question du droit des travailleurs à peser pleinement sur le gouvernement de l'entreprise à laquelle ils contribuent, c'est-à-dire non seulement de leur permettre de peser sur les moyens de l'activité, mais également sur les fins.

19 I. Ferreras, Gouverner le capitalisme ? Pour le bicamérisme économique. Paris. Presses universitaires de France, 2012.

20 Dans la filiation des réflexions classiques de Pizzorno sur l'« échange politique », on s'appuie ici sur la distinction, au plan analytique et non pratique, proposée avec Jean De Munck, des trois composantes de ce que nous appelons l'« échange démocratique », à savoir : délibération, marchandage (bargaining) et expérimentation (J. De Munck, I. Ferreras. «The Democratic Exchange as the Combination of Deliberation, Bargaining, and Experimentation ») in J. De Munck, Cl. Didry, I. Ferreras et A. Jobert (sld.), Renewing Democratic Deliberation in Europe. The Challenge of Social and Civil Dialogue. July 2012, Oxford-Brussels, Peter Lang Press, pp. 149-169.

21 II reste à démontrer que le mouvement des « entreprises libérées » (B. Carney, I. Getz, 2012. Liberté \& Cie. Paris. Fayard, 2012) et autres pratiques dites de Responsabilité sociale de l'entreprise dépassent l'enrôlement des salariés dans la gestion, au profit toujours indiscutable des propriétaires des parts de la société. 


\section{V - COMMENT OPÉRER LA TRANSITION?}

Pour opérer cette transition, nos sociétés occidentales sont chacune passées par un « moment bicaméral $»^{22}$. Plaçant l'entreprise dans histoire politique longue, on peut envisager une issue au fait que les investisseurs en travail se trouvent dans une situation d'incapacité à peser sur le gouvernement de l'entreprise, droit qui a été par ailleurs reconnu aux apporteurs de capital via la société anonyme. Deux parties «constituantes ${ }^{23}$ font l'entreprise : il faut en effet deux types d'investissement pour qu'une entreprise puisse mener un projet à bien, capital et travail. L'apport en capital a été reconnu et valorisé comme digne d'une structuration institutionnelle, l'investissement en travail attend... Or, la présence d'un Comité d'entreprise ou d'un Conseil d'entreprise constitué de représentants élus par les travailleurs dans la seconde moitié du XXème siècle dans certains pays européens dont le France, jusque l'existence de la Mit-bestimmung (co-détermination) en Allemagne en passant par la constitution de Conseils d'entreprise européens témoignent de l'intuition qu'une représentation des travailleurs dans le gouvernement de l'entreprise, via une structuration institutionnelle, est légitime et nécessaire. II convient de cesser de ne privilégier que l'une des deux logiques fondatrices de l'entreprise (la logique instrumentale du capital à l'heure de la financiarisation de l'économie mondialisée sur la rationalité politico-expressive des salariés), qu'une seule de ses deux parties constituantes, les apporteurs en capital, au détriment de l'autre, les investisseurs en travail. Relire l'histoire du bicamérisme politique, la théorie politique et la théorie du droit qui la commente, permet de mesurercombien la reconnaissance de cette logique duale se trouve au cœur même de l'avancée des sociétés démocratiques et de la solution qu'elles ont trouvée pour sortir du despotisme et amorcer le processus de démocratisation de nos sociétés.

Poursuivre cette histoire longue dans le contexte de l'entreprise, c'est reconnaître que le gouvernement de l'entreprise, son pouvoir exécutif, peut ainsi être repensé comme responsable, non plus devant les seuls apporteurs en capital (via le Conseil d'administration), mais devant les deux corps constituants de l'entreprise, les apporteurs en capital et les investisseurs en travail, qui, ensemble, font l'entreprise. Dans ce régime bicaméral, les deux groupes sont représentés au travers de leurs élus au sein de deux Chambres. Celles-ci garantissent une représentation propre et forçant les deux corps constituant l'entreprise à coopérer pour peser ensemble en tant que pouvoir législatif. Responsable devant chacune des deux Chambres, réunies de préférence conjointement afin de maximiser l'apprentissage des logiques propres et leur impact délibératif, le top management élu par les deux chambres devient alors chargé de trouver des compromis productifs pour le développement de l'entreprise dans l'intérêt des deux parties, et non plus d'une seule. En effet, une majorité (50\%+1 voix) est exigée dans chacune des Chambres pour qu'une loi

22 I. Ferreras, Gouverner le capitalisme ? Pour le bicamérisme économique. Paris. Presses universitaires de France, 2012. Du même auteur, Firms as Political Entities. Saving Democracy through Economic Bicameralism. Cambridge/New York City: University Press, 2017.

23 I. Ferreras, Gouverner le capitalisme ? Pour le bicamérisme économique. Paris. Presses universitaires de France, 2012. 


\section{DROIT DU TRAVAIL, DROITS CIVILS ET POLITIQUES}

soit adoptée ${ }^{24}$. Chacune des deux parties détient ainsi un droit de veto sur les décisions fondamentales de la vie de l'entreprise ${ }^{25}$.

Envisager d'amener à maturité l'intuition critique qui anime l'histoire du droit du travail est un enjeu capital. Des institutions chèrement gagnées sont en train d'être défaites aujourd'hui, les libertés syndicales étant en recul dans la plupart des pays occidentaux avec l'impact que l'on sait sur le développement de plus en plus inégalitaire de nos sociétés ${ }^{26}$, et son effet en retour sur le comportement électoral ${ }^{27}$ destructeur de nos sociétés démocratiques. L'intuition de la citoyenneté au travail, ainsi résumée en introduction aux lois Auroux de 1981 : « citoyens dans la cité, les travailleurs doivent l'être aussi dans leur entreprise " est à prendre au sérieux aujourd'hui plus que jamais si nous voulons sortir de la contradiction capitalisme/démocratie, en Europe, via plus de démocratie, et non pas plus de despotisme capitaliste. Et le droit du travail, soutenant la possibilité de l'égale dignité des investisseurs en travail, doit s'envisager aujourd'hui comme occupant une place première - de combat - au sein des droits civils et politiques.

24 A la grande différence de la Mit-Bestimmung qui reste un système monocaméral et dépend d'une simple majorité dans une chambre unique. Le banc patronal obtient dans ces conditions facilement une majorité. Pour plus de détails sur la différence entre ces schémas de gouvernement, voir (I. Ferreras, Firms as Political Entities. Saving Democracy through Economic Bicameralism. Cambridge/New York City, University Press, 2017).

25 En termes de faisabilité pratique, on peut imaginer qu'une société qui estimerait souhaitable d'encourager ce modèle de gouvernement démocratique, estimant qu'il correspond mieux à ses valeurs que le gouvernement despotique du capital, mette en place un régime fiscal favorable pour ce type de sociétés. On aurait déjà vu donner aux entreprises des incitations fiscales pour de moins bonnes raisons que celle-ci...

26 Voir F. Jaumotte, C. Osorio Buitron, "Inequality and Labor Market Institutions », International Monetary Fund Research department, IMF Staff Discussion Note, SDN/15/14, July 2015, 31 pp.

27 Voir l'étude de Coutrot (Th. Coutrot, Thomas, 2018, "Travail et bien-être psychologique» (http://dares.travail-emploi.gouv.fr/IMG/pdf/travail_et_bien-etre_tc_vd_2.pdf). DARES, Etude $n^{\circ} 217$, mars 2018), qui démontre pour le cas français le lien entre conditions aliénantes de mise au travail et vote pour l'extrême droite ou abstentionnisme.

\section{ISABELLE FERRERAS}

Maître de recherches du FNRS-Fonds de la recherche scientifique (Bruxelles), Professeure à I'Université de Louvain (Belgique), membre permanent du CriDIS (Centre de recherches interdisciplinaires Démocratie Institutions, Subjectivité), de l'Institut IACCHOS et Senior Research Associate du Labor and Worklife Program, Harvard Law School, de I'université d'Harvard (Cambridge MA, USA).

Thèmes de recherche : Sociologie des services, transformations du travail et de l'entreprise, action syndicale, théorie politique de l'entreprise, avenir de la démocratie.

\section{Publications :}

J. Charles, I. Ferreras, « La Citoyenneté au travail. Enjeu pour les organisations et la sociologie » in Travail et Care comme expériences politiques, M. de Nanteuil et L. Merla (sld). Louvain-laNeuve, Presses universitaires de Louvain, 2017, pp. 165-78.

$\sim$ H. Landemore, I. Ferreras, "In Defense of Workplace Democracy: Towards a Justification of the Firm-State Analogy », Political Theory, 2016, Vol. 44(1) 53-81. 